\title{
Transatlantica
}

Revue d'études américaines. American Studies Journal

\section{Annick Duperray. Paul Auster, les ambiguïtés de la} négation.

Paris : Editions Belin, 2003. 126 pages.

\section{François Gavillon}

\section{OpenEdition}

\section{Journals}

Édition électronique

URL : http://journals.openedition.org/transatlantica/867

DOI : $10.4000 /$ transatlantica.867

ISSN : 1765-2766

Éditeur

AFEA

\section{Référence électronique}

François Gavillon, « Annick Duperray. Paul Auster, les ambiguïtés de la négation. », Transatlantica [En

ligne], 1 | 2005, mis en ligne le 23 avril 2006, consulté le 29 avril 2021. URL : http://

journals.openedition.org/transatlantica/867 ; DOI : https://doi.org/10.4000/transatlantica.867

Ce document a été généré automatiquement le 29 avril 2021.

\section{c) (i) $\Theta$}

Transatlantica - Revue d'études américaines est mis à disposition selon les termes de la licence Creative Commons Attribution - Pas d'Utilisation Commerciale - Pas de Modification 4.0 International. 


\section{Annick Duperray. Paul Auster, les ambiguités de la négation.}

Paris : Editions Belin, 2003. 126 pages.

François Gavillon

1 Avec l'ouvrage d'Annick Duperray, Paul Auster, les ambiguités de la négation, la collection « Voix américaines » s'est enrichie d'un nouveau titre. En un peu plus d'une centaine de pages - quatre chapitres et un épilogue, augmentés d'une chronologie et d'une bibliographie sélective, outils qui seront fort appréciés des lecteurs d'Auster (les nouveaux, comme les habitués) - , Annick Duperray offre un tableau précis et complet de

l'œuvre austérienne.

Le premier chapitre, "l'homme ou l'œuvre», met le doigt sur l'une des questions fondamentales de cet univers: la tension entre biographie personnelle et fiction. Annick Duperray fait clairement voir qu'une donnée essentielle de l'écriture austérienne est le hiatus qui existe entre moi d'auteur et moi biographique ou social. S'appuyant sur les notions de "(dé)négation » et de "mort de l'auteur ", Annick Duperray montre combien névralgique chez Auster est le point de rencontre entre biographie et autographie. L'œuvre est ce lieu hybride où sont affrontés l'auteur et son portrait in absentia, auteur déréalisé, désœuvré à la fois dans la réalité et dans la fiction. C'est aussi le lieu des paradoxes et des dualités où se côtoient la parabole et l'histoire personnelle, où se lit la présence simultanée du réel et de l'imaginaire. La section intitulée " parcours » (pages 19-28) donnera par ailleurs une parfaite vue d'ensemble de la vie d'Auster, des différents genres dans lesquels il s'est illustré, ainsi que des dates des productions artistiques principales.

Le deuxième chapitre retrace le parcours poétique de l'écrivain avant qu'il ne se consacre principalement au roman. Depuis Unearth (1975) jusqu'à Facing the Music (1988), Annick Duperray analyse le détail de la production où s'élabore une poétique dans laquelle on discerne l'influence de Charles Reznikoff, de George Oppen, l'intérêt pour John Ashbery, mais aussi pour Mallarmé, Rimbaud, Dupin. L'étude d'Annick 
Duperray fait voir l'attention que porte la poétique austérienne au processus de perception, à la rencontre phénoménologique entre moi subjectif et réalité empirique. Elle fait apparaître, au-delà des caractéristiques de minimalisme, de concision, et souvent d'hermétisme, les différentes phases de la production poétique, tour à tour sombre et dense (Unearth, Fragments from Cold) ou au contraire ouverte (Wall Writing) qui mènent au dialogisme des textes à venir. Le texte hybride White Spaces (1980) est au croisement de deux modes d'expression, poésie et prose. Ce texte annonce un ouvrage majeur, The Invention of Solitude (1982), analysé ici dans ses enjeux véritables. Dans la première partie, «Portrait of an Invisible Man », « l'acte d'écriture devient en quelque sorte une reconnaissance de paternité à rebours [...] le fils [Paul Auster] entreprenant « de recréer (ou inventer) le père par la force du souvenir et le pouvoir des mots ». La seconde partie, "The Book of Memory", fait l'objet d'une analyse formelle, chronologique, thématique. Est en particulier souligné le caractère simultané, sinon synonyme, de l'écriture, de la mémoire, de la présence de soi et de l'autre. Le point commun à plusieurs romans austériens est la ville. Le troisième chapitre s'attache à l'analyse de ce décor urbain. Espace concret fait de briques, de rues et de façades, mais aussi espace déréalisé, virtuel qui figure tout autant un labyrinthe géographique qu'une errance au cœur de la psyché. Annick Duperray montre comment les héros de la Trilogie new-yorkaise, sujets clivés victimes d'une folie ordinaire, sont des "personnageslimites", oscillant entre rigidité psychique et abandon, fantasme d'absolu et clochardisation. Les romans de la Trilogie, faux romans policiers, « parce qu'ils relèvent du questionnement plus que de l'interprétation, de l'énigme plus que de la détection ", oscillent eux-mêmes entre consignation scrupuleuse des faits et aphasie, lucidité et démence, entre autorité et pseudonymie, fiction et métafiction. La ville de New York dans la Trilogie, puis la Ville dans In the Country of Last Things sont des lieux concentriques, labyrinthiques où le rêve original de pureté et d'absolu s'effondre. On erre dans la ville comme on s'abîme dans un lieu de mémoire-individuelle ou collective - névralgique, infernal. In the Country of Last Things bruit des horreurs de l'Holocauste, mais aussi de la détresse et de la déréliction qui hantent les mégalopoles d'aujourd'hui. Pourtant Anna Blume est une résistante. Son travail d'écriture et de mémoire est acte de foi en un possible après. « Un rêve de résurrection hante le récit d'Anna » et là où l'espoir demeure, l'humanité ne meurt pas tout à fait. Il faut imaginer Anna heureuse.

Avec Moon Palace, The Music of Chance et Leviathan, le décor urbain éclate et les fictions prennent le large. C'est cet élargissement de l'horizon qu'aborde le quatrième chapitre. Annick Duperray fait d'emblée remarquer que l'ouverture territoriale s'inscrit dans une dimension politique. Moon Palace comme Leviathan font le récit de trajectoires individuelles sur fond d'Histoire nationale. Les héros sont des idéalistes, des quêteurs d'absolu (symbolisé par la lune, inaccessible objet). Mais les rêves de retour aux origines, de perfection créatrice, d'harmonie au sein d'une nature prélapsarienne sont frappés d'inanité. La Lune est bien atteinte en 1969, mais entre-temps, le rêve s'est dénaturé, l'Amérique s'est dévoyée. Le "rêve d'Amérique " s'est dégradé en "rêve américain ", dit Annick Duperray, citant Marc Chénetier. L'étude fait également apparaître la dimension subversive à l'œuvre dans l'écriture: Moon Palace courtise et détourne les genres du roman victorien et du roman picaresque. Il y a dans les efforts des trois générations de héros un caractère donquichottesque qui bascule parfois dans l'absurde, annonçant par là The Music of Chance. Ce roman a, selon Annick Duperray, la 
force du conte. Conte moral, symbolique, allégorique. Le parcours du héros est un parcours initiatique au terme duquel celui-ci découvre que « la liberté n'est rien d'autre que la connaissance de la nécessité». Le roman, moins romance picaresque que parabole, fait entendre une note existentialiste. Qu'est-ce que la liberté ? Comment donner un sens au monde ? Comment effacer le sentiment de la dette devant « la perte d'autorité » et "l'indignité des pères»?

Dans son épilogue, Annick Duperray passe plus rapidement sur les textes de la dernière période qu'elle juge ludiques et moins convaincants. A la recherche de nouvelles modalités créatrices, les romans laissent parfois la place aux histoires vraies, autobiographie (Hand to Mouth) ou compilation de récits allogènes (True Tales of American Life). Quant aux romans de cette période, Mr Vertigo, Timbuktu, The Book of Illusions, Annick Duperray les voit marqués du sceau de la dérision et de la nostalgie, de l'humour et de la mélancolie. De l'œuvre romanesque dans son ensemble, on pourra sans doute dire qu'elle est postmoderne si l'on tient compte de son caractère autographique et métafictionnel, de l'importance accordée à l'interaction du langage et de la subjectivité, mais, Annick Duperray le rappelle, les expérimentations narratives qui comportent de tels élément ne sont pas inédites. Cervantès, Shakespeare, Joyce (au moins) font figure d'illustres prédécesseurs. C'est l'un des grands mérites de l'analyse d'Annick Duperray que de mettre en évidence le fait que l'écriture austérienne, si elle est faite de jeu et d'ironie, s'enracine aussi dans le questionnement et l'expérience intimes.

L'ouvrage est rendu précieux, en outre, par les notations nombreuses et précises des circonstances biographiques, historiques, chronologiques dans lesquelles l'œuvre naît. Chaque chapitre est introduit par un bref exposé des points caractéristiques qui sont analysés ensuite œuvre par œuvre. Claire et précise, détaillée et globale à la fois, l'étude d'Annick Duperray remplit à merveille sa mission : dévoiler les enjeux profonds d'une œuvre dans laquelle le lecteur pourra s'orienter sans pour autant que ne lui soit ravi le plaisir des textes.

\section{INDEX}

Thèmes : Recensions

\section{AUTEUR}

\section{FRANÇOIS GAVILLON}

Université de Bretagne Occidentale, Brest 